\title{
Laboreal
}

Volume $5 \mathrm{~N}^{\circ} 2$ | 2009

Varia

\section{Análisis y estructura del trabajo}

Análise e estrutura do trabalho

Analyse et structure du travail

Analysis and structure of work

\section{Jean-Marie Faverge}

Traductor. Jesús Villena

\section{OpenEdition}

\section{Journals}

Edición electrónica

URL: http://journals.openedition.org/laboreal/9913

DOI: 10.4000/laboreal.9913

ISSN: 1646-5237

Editor

Universidade do Porto

\section{Referencia electrónica}

Jean-Marie Faverge, «Análisis y estructura del trabajo », Laboreal [En línea], Volume 5 N² | 2009,

Publicado el 01 diciembre 2009, consultado el 24 septiembre 2020. URL : http://

journals.openedition.org/laboreal/9913; DOI : https://doi.org/10.4000/laboreal.9913

Este documento fue generado automáticamente el 24 septiembre 2020.

\section{c) (i) (8)}

Laboreal está licenciado com uma Licença Creative Commons - Atribuição-NãoComercial 4.0 Internacional. 


\title{
Análisis y estructura del trabajo
}

\author{
Análise e estrutura do trabalho \\ Analyse et structure du travail \\ Analysis and structure of work
}

Jean-Marie Faverge

Tradución : Jesús Villena

\section{REFERENCIA}

Artículo original : Faverge, J.-M. (1952). Analyse et structure du travail. Bulletin du Centre d'Études et de Recherches Psychotechniques (CERP), nº1, janvier-juin.

1 El estudio psicotécnico del trabajo comporta los procedimientos analíticos destinados a aislar los elementos importantes. El análisis conduce a la localización de esos elementos para describirlos mejor y rechazar todo lo que es accesorio. Sin embargo, los psicólogos saben lo peligrosa que puede ser la aplicación del método analítico en la observación de comportamientos humanos. Esta aplicación se arriesga a destruir ciertas estructuras, ciertas unidades, ciertas moléculas si nos remitimos a una analogía con la química. El inventario de fragmentos así obtenidos no permite dar cuenta del comportamiento real y sólo puede dar lugar al verbalismo. Es lamentable que estas ideas, desarrolladas particularmente por la psicología de la forma, no hayan sido introducidas en los diferentes ámbitos en los que se practica el análisis del trabajo, como vamos a demostrar a través de diversos ejemplos.

\section{El análisis de los tiempos en el trabajo}

2 Se sabe que en este terreno, en la actualidad, se manifiesta una tendencia que tiene como objeto sustituir los métodos directos de cronometraje por métodos analíticos. Las dos técnicas más conocidas son americanas : la técnica Q. S. K (Quick, Shea y Koehler) y la más reciente técnica M. T. M. (Method time measurement) de Maynard. 
Utilizan la siguiente metodología :

1. Investigar elementos universales del trabajo, es decir, elementos a partir de los cuales podemos analizar cualquier tarea. Los antecedentes están en Gilberth con sus therbligs.

2. Considerar el tiempo utilizado para hacer un trabajo como una suma de los tiempos elementales que componen el trabajo.

4 El riesgo de estos métodos es precisamente el de fragmentar las estructuras en el interior de las cuales los tiempos no se suman. Puede darse un gran número de ejemplos de aquello que denominamos el cambio de la secuencia de los therbligscuando se aumenta la rapidez del trabajo. Este cambio consiste en la aparición de superposiciones entre los therbligs $y$ las operaciones que se realizadan simultáneamente.

5 Recordemos un ejemplo estudiado por Gérard de Montopellier : Pidió a un sujeto que tocara los cuatro vértices de un rectángulo de forma ininterrumpida. Constató que, cuando la velocidad aumenta, el gesto se redondea y el movimiento de la mano describe una especie de elipse. Hay, por lo tanto, superposición de los gestos porque la naturaleza elíptica del trayecto indica que, en el momento en el que se realiza un toque, ya se están preparando los toques siguientes.

6 Algunos especialistas del estudio de los tiempos se dieron cuenta del peligro e investigaron los medios para probar la hipótesis de que los elementos del trabajo obtenidos son, de hecho, unidades . Formularon el siguiente criterio :

"El tiempo de un elemento se mantiene igual, independientemente de los elementos que lo preceden o que vienen después en la operación"

7 Según los autores, la ventaja del método M.T.M. sobre el más antiguo método Q.S.K. es, precisamente, el hecho de tener en cuenta elementos que obedecen al criterio anterior.

8 La diferencia esencial entre los dos métodos es que el Q.S.K. define los movimientos de cada parte del cuerpo (brazos, piernas, tronco, dedos, manos, pies) mientras que el M.T.M. define los gestos a partir de su objeto (alcanzar, mover, volverse, agarrar, "posicionar", desprender).

9 Como dijo M. Louden en su informe del último Congreso Internacional de la Organización Científica del Trabajo de Bruselas, "el método M.T.M. no contiene sólo los baremos que determinan el tiempo normal para cada tipo de movimiento dado en condiciones variables, sino que formula también leyes de encadenamiento de estos gestos".

10 Por otro lado, actualmente se investiga en una universidad americana el tiempo de un elemento, coon vistas a estudiar los efectos de los elementos adyacentes.

11 Indiquemos así mismo que los belgas han recuperado los elementos del método Q.S.K. para analizar el trabajo con el objetivo de readaptar a deficientes y que ese método puede terminar siendo inútil, por las razones que estamos desarrollando.

12 Se observa así que las técnicas modernas tienen la preocupación, nacida tras las numerosas desilusiones resultantes de la experiencia, de tener en cuenta la estructuración del trabajo en el estudio de los tiempos y de no hacer una separación aleatoria de las operaciones. Señalemos que en el análisis del trabajo, prácticamente, la noción de estructuración se mezcla casi con la de significación. Un elemento, tal como 
esperar, volverse, tienen un significado y se corre el riesgo de hacer una separación inadecuada si aislamos el gesto de su objeto.

\section{La aparición de las estructuras en el aprendizaje}

13 EL que aprende un trabajo observando se encuentra con un fenómeno bastante sorprendente: ante la primera dificultad importante, pasa por un período de desorientación a veces largo en el que no progresa y se pregunta si algún día llegará a hacerlo bien.

14 Es esto lo que nos ocurrió cuando aprendimos a poner ladrillos, no conseguimos poner nuestros ladrillos en la posición correcta, se quedaban la mayor parte de las veces inclinados hacia nuestra lado y las juntas verticales se quedaban casi vacías. En ese momento, como aprendices concienzudos, dirigimos nuestra atención a todos los puntos señalados por el monitor : mortero suficientemente mojado pero no demasiado, cantidad de mortero a recoger con la paleta, lugar donde debemos depositarlo, forma de extenderlo, la forma que tiene después de extenderlo, colocarlo contra el ladrillo adyacente, forma de colocar el ladrillo, siguiendo la línea y comprimiendo suficientemente el mortero para hacer una junta horizontal con un espesor adecuado, etc... ¡Y mientras nos esforzamos por hacer todo esto, el mortero se ha secado !... Estábamos en el famoso período de estancamiento descrito por los psicólogos. Después, un buen día, un poco como dándonos cuenta bruscamente de que nuestros bebés andan o hablan, constatamos que posicionamos bien y rápidamente los ladrillos y que dejábamos de lado todas las recomendaciones, limitándonos a colocar nuestro ladrillo de forma adecuda, orientándolo por un vistazo al canto de la derecha, formado por la arista vertical de y la arista horizontal del ladrillo de la fila de abajo.

Conocemos fenómenos análogos que ocurren durante el aprendizaje de la lectura, de la dactilografía o del código morse. Al principio aprendemos las letras, después las palabras de una forma global y en seguida unidades compuestas de palabras. Las estructuras aparecen así en el transcurso del aprendizaje y sería absurdo no reconocerlas o destruirlas en el análisis psicotécnico del trabajo. De este modo, cuando aprendemos una profesión para comprenderla, nuestras primeras observaciones son frecuentemente poco importantes, sea cual fuere el interés que prestamos en su observación, porque la naturaleza del trabajo cambia enseguida y sólo corresponden a un momento del aprendizaje que se esfuma rápidamente .

16 Presentamos a continuación una ilustración en las que pueden verse los tiempos medios de inserción de 40 perforaciones de una tarjeta [1], obtenidos a través de la filmación en cámara lenta de catorce mecanógrafos. Los números $6-12-7-15$ corresponden a palabras o números transcritos y señalaremos los puntos que son visibles en las articulaciones entre esos conjuntos y los que reflejan la existencia de estructuras en la pulsación. 
Figura 1

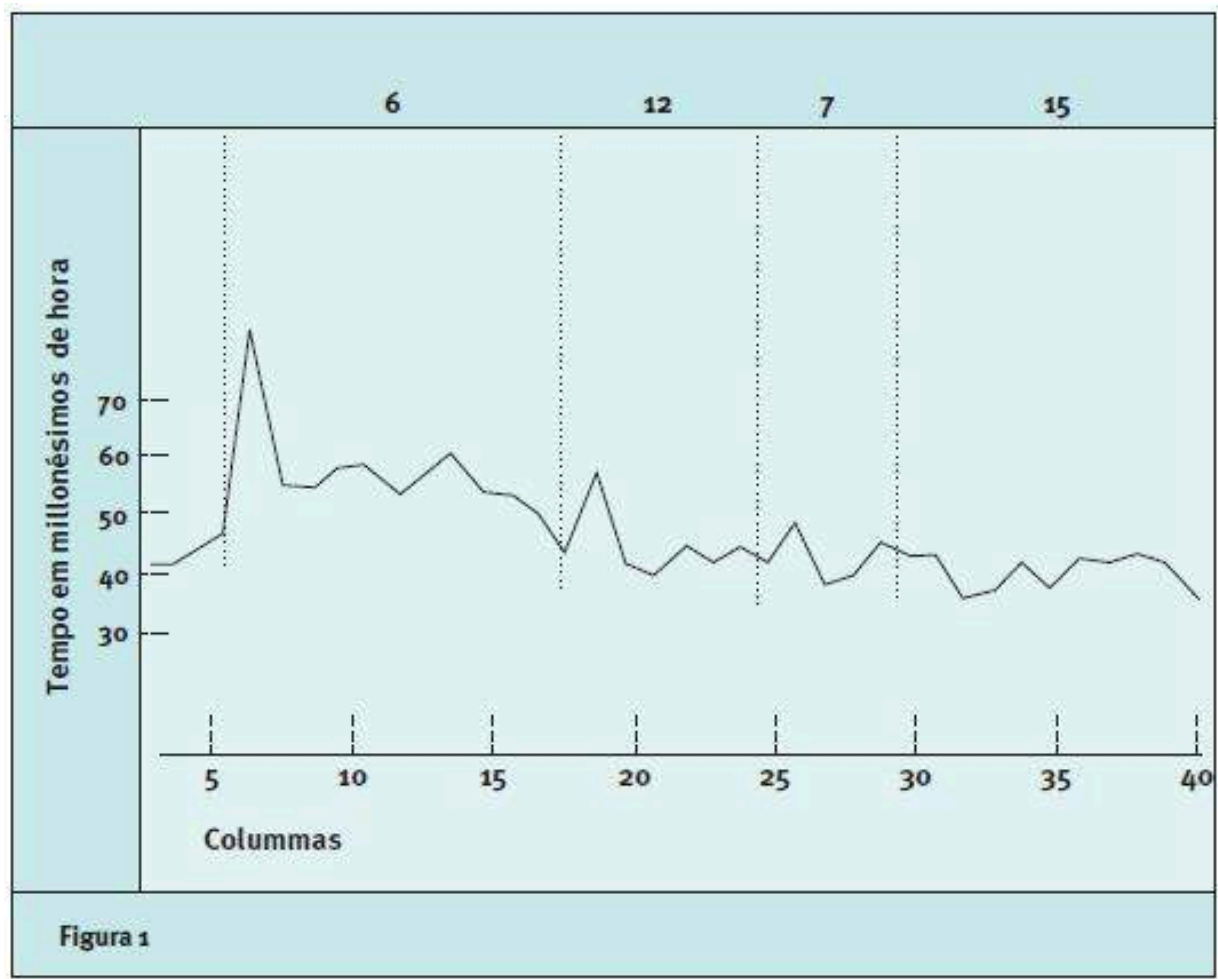

\section{La crítica al análisis en términos de aptitudes}

Sabemos que es habitual encontrar monografías en las que aparecen perfiles que contienen con frecuencia "aptitudes". El autor de tales monografías adopta una lista de términos, que son sus puntos de referencia $\mathrm{y}$, de una forma más o menos sistemática, los revisa teniendo en cuenta el puesto de trabajo considerado. A veces evalúa incluso el grado de cada aptitud.

Vemos, por ejemplo, un perfil-tipo de una operadora de máquina de camisas de hombre, que utiliza las aptitudes del Dr. Carrard (véase fig. 2). 
Figura 2

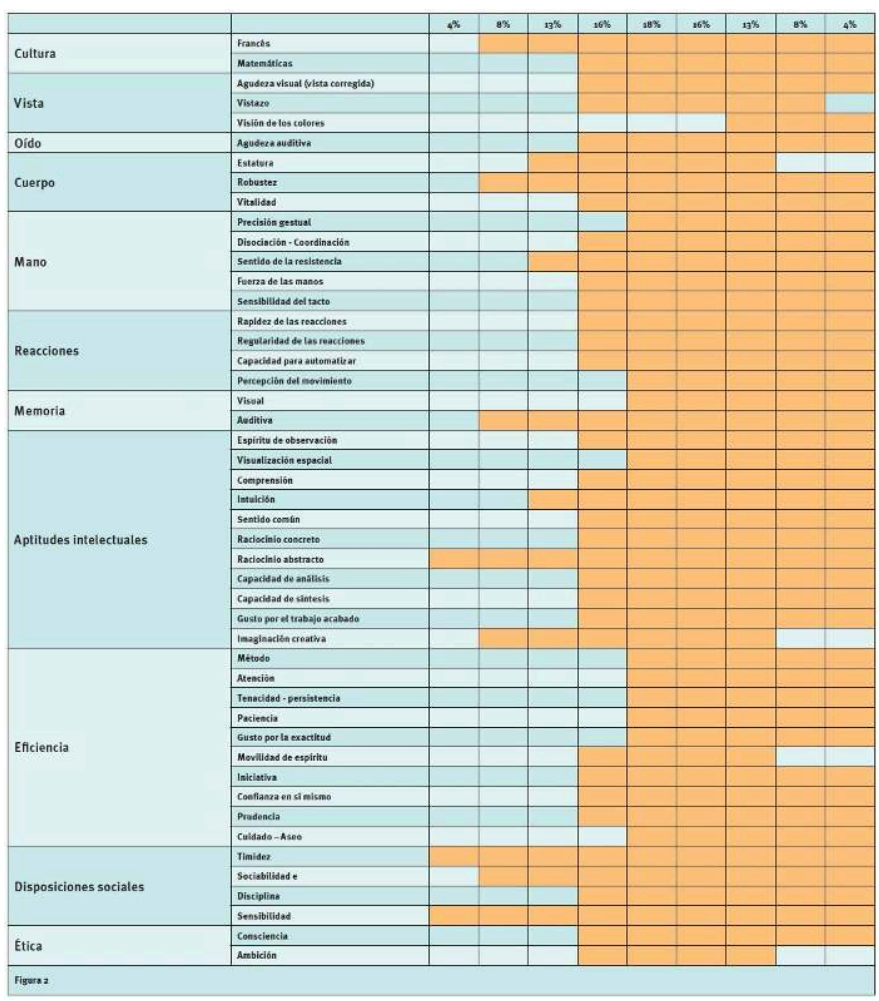

19 No vamos a detenernos para sonreir al comprobar la preocupación por la precisión en los niveles de la aptitud, no insistiremos en conocer la población de referencia en la cual los porcentajes son válidos, ni cuestionaremos si las variables son cuantitativas y apreciables en grados, etc... examinaremos simplemente las dos rubricas "vistazo" y "precisión gestual" que el autor considera como particularmente importantes para este puesto de trabajo.

21 El vistazo - Es bastante difícil de precisar lo que entendemos por vistazo. En un gran número de profesiones, los profesionales dicen "es necesario capacidad para echar un vistazo". Es así, por ejemplo, en la construcción civil, y nosotros entendemos entonces, en general, que el vistazo tiene por objetivo permitir ver si las aristas de los muros son paralelas, si las fachadas tienen defectos de verticalidad, si las reglas están derechas, etc... el ojo debe estar colocado en una buena posición, bien determinada; si se colocado un poco de lado, aún vemos, pero hacemos entonces una simple apreciación y no obtenemos la misma precisión.

Por otro lado, llamamos frecuentemente "vistazo" a la apreciación con exactitud de una longitud o de un ángulo. Así, numerosas pruebas de esa manara de mirar consisten en cuestiones donde se pide al sujeto que indique la longitud o el ángulo de entre cinco o seis figuras que son exactamente igual a un modelo. Esto es evidentemente muy diferente del vistazo que echa el albañil. Entonces, que quiso el decir el autor al darse cuenta de que el vistazo debe superar el $25 \%$ de su población objeto de estudio? Probablemente, pensó en los índices visuales que guían el trabajo de la operadora de la máquina. Esto representa aún otra cosa, como vemos a través de la lectura del artículo de Mlle Saint-Just, en las páginas siguientes. 
Veamos pues las operaciones de trabajo habituales que recurren al "vistazo".

- Evaluar con elevada precisión al leer un dispositivo

- Verificar o reparar los errores de un montaje

- Centrar rápidamente

- Controlar defectos a partir del aspecto

- Estimar los espacios, volúmenes, etc...

- Estimar las distancias

- Evaluar el aspecto de piezas en movimiento

- Ver y evaluar detalles huidizos, etc...

\section{Precisión gestual}

Podemos repetir aquí lo que se ha dicho a propósito del vistazo. Así, la expresión "precisión gestual" se refiere tanto al gesto de la mano izquierda que se apoya sobre el tejido y, gracias a un juego de dedos y al punto de apoyo de la muñeca, hace girar el tejido de forma adecuada y continua cuando la modista cose según una línea curva, como al gesto preciso y rápido de la mano izquierda en la soldadura de hierro, gesto a través del cual, cuando el molde está suficientemente caliente, se introduce la cantidad exacta de soldadura.

Se han hecho múltiples estudios factoriales sobre las aptitudes motoras. Una síntesis de esos estudios demostraría que sólo un factor se ha establecido correctamente (ver por ejemplo Seashore). Es un error tratar como un factor de precisión aquel en el que se saturan las pruebas sobre las que se pide realizar una tarea, como el hecho de salir de un lugar preciso o de un trayecto se constituye un error (por ejemplo en el test del tornero). No se trata aquí de precisión en los movimientos o en las conductas sino en el resultado del trabajo. No es una "aptitud" que da unidad a las pruebas, es la significación del resultado que se quiere alcanzar. En el mismo orden de ideas podemos recordar lo profunda que fue la crisis que atravesón la psicología tradicional de las capacidades cuando quisieron someter esos conceptos a la experimentación. El análisis factorial nació en el momento de esa crisis, y veamos lo que produjo en el ámbito mental :

Aparecieron tres factores sobre los cuales el acuerdo fue general, porque fueron encontrados por todos los experimentadores. El resto de factores son más criticados, y que daban cuenta de una parte mucho más débil de la variancia y varían de un autor para el otro.

Esos tres factores principales, que por otro lado no se esperaban, pueden llamarse : verbal, numérico y gráfico o espacial. Se han definido respectivamente por las pruebas de soporte verbal, numérico o gráfico. Estamos lejos de las capacidades ligadas a los mecanismos del espíritu: juicio, raciocinio, atención, etc ... y nos vemos obligados a pensar que el análisis factorial reagrupó las tareas en función de su significación.

De este modo podemos avanzar que las famosas aptitudes no pueden ser aisladas de ciertas estructuras, que haciéndolo destruimos las moléculas de las que hablamos y que un análisis del trabajo en términos de aptitudes es puro verbalismo.

Para terminar vamos a contar una desgracia que nos ocurrió recientemente cuando estudiábamos el aprendizaje de la soldadura con el soplete. Después de las 
observaciones hechas mientras aprendíamos a soldar, emitimos la siguiente hipótesis : Las dificultades del trabajo son:

1. La vigilancia de la anchura del baño de fusión.

2. La ejecución de pequeños movimientos alternativos (amplitud de 1 a $2 \mathrm{~mm}$ ) con la extremidad de una barra de soldadura con la mano izquierda. Esos pequeños movimientos debían ser muy regulares, preferentemente con un ritmo en forma de dientes de sierra.

Efectivamente, gracias al montaje de un osciloscopio catódico en el puesto de trabajo constatamos, en una sesión de aprendizaje, que el monitor y los buenos alumnos realizaban gestos regulares y finos, como si fueran teclas. En los malos alumnos observamos irregularidades y barra sumergida mucho menos veces en el área de fusión. Construimos entonces un aparato destinado a reproducir el gesto pero donde, obviamente, no hay zona fusión. El sujeto debía tocar con la extremidad de una barra de forma alterna los dos bordes de una arista, siguiendo los movimientos de un metrónomo. Constatamos que el monitor no seguía la cadencia, que la extremidad de su barra temblaba al punto de no conseguir pasar la prueba, que los buenos alumnos no se distinguieron de los malos en la ejecución y en la regularidad de los gestos.

31 Concluimos que habíamos sido una vez más víctimas de esa noción de aptitud, enraizada en nosotros, aptitud para efectuar movimientos finos con la mano izquierda que no representa nada sin el significado de la tarea propuesta.

\section{NOTAS}

1. Se refiere a la perforación de las tarjetas que requerían las primeras grandes computadoras (N.del T.) 